\title{
THE CLINICAL PRESENTATION OF CHILDREN WITH TUMOURS AFFECTING THE ANTERIOR VISUAL PATHWAYS
}

\author{
J. SUHARWARDY ${ }^{1}$ and J. ELSTON ${ }^{2}$ \\ Oxford
}

\begin{abstract}
SUMMARY
Purpose: To review the ways in which children with tumours affecting the anterior visual pathways present and to determine the extent of visual loss at presentation and the visual prognosis after treatment.

Methods: A retrospective review of the 17 children (age 1-13 years at presentation) referred to a specialist paediatric oncology unit. Ten children had extrinsic tumours affecting the chiasmal area whilst 7 had intrinsic gliomas affecting the optic nerve and/or chiasm. Patients were followed up for up to 13 years (median 2 years).

Results: The children studied presented with a variety of symptoms including headaches, lethargy and growth failure. Despite the clinical finding of marked visual loss in several cases, visual failure was not prominent amongst the presenting symptoms. After surgical intervention and other treatment vision stabilised or improved; eyes with no perception of light for short periods could regain substantial vision.

Conclusion: Children with progressive visual deterioration due to tumours do not readily verbalise their difficulty and may have profound loss at presentation. Standard clinical tests of vision will identify the extent of damage. Visual prognosis depends on the underlying pathology and length of history but prompt treatment may lead to substantial improvement in vision.
\end{abstract}

Tumours in children affecting the anterior visual pathways form a significant proportion of childhood primary brain neoplasms. ${ }^{1,2}$ These tumours are divided into intrinsic tumours of the optic nerve, chiasm and tract and extrinsic tumours compressing the visual pathway at some stage between the orbital part of the optic nerve and anterior optic tract.

The clinical presentation depends on the position

From: ${ }^{1}$ University Department of Ophthalmology, Manchester Royal Eye Hospital, Manchester; ${ }^{2}$ Oxford Eye Hospital, Oxford, UK.

Correspondence to: Mr J. Elston, The Oxford Eye Hospital, Walton Street, Oxford OX2 6AN, UK. of the tumour. Optic nerve tumours may present with visual loss or proptosis whilst intracranial tumours involving the optic chiasm may present with visual failure. Intracranial tumours can also present with a wide variety of signs and symptoms, the most common of which are headaches either associated with increased intracranial pressure or secondary to meningeal stretching, endocrine dysfunction or evidence of focal neurological disturbance.

We have been concerned at the extent of visual loss on presentation in a number of children with tumours involving the anterior visual pathways. We have therefore analysed the visual findings at presentation and follow-up, together with any other presenting symptoms and signs, in 17 children who presented to our unit.

\section{PATIENTS, MATERIALS AND METHODS}

We reviewed the clinical records of the last 17 children diagnosed with a tumour affecting the anterior visual pathways. They were divided into two groups: 10 had tumours of the suprasellar region involving the chiasm whilst 7 had intrinsic tumours of the optic nerve and chiasm.

Patients were primarily under the care of the regional specialist paediatric oncology unit and all had an ophthalmological assessment. This was generally done at the time of initial presentation although occasionally it was done later and in certain circumstances after initial neurosurgical intervention.

A full ophthalmic examination was attempted in all cases and included visual acuity and visual field testing, which in most cases was possible with a Snellen chart and Goldmann field respectively. We assessed the optic discs, colour vision (with an Ishihara chart) and pupil responses, although in certain cases a more limited examination was necessary due to the medical condition of the child. All patients had MRI scans by an experienced neuroradiology department. 
Table I. Presenting symptoms with their duration in children with suprasellar tumours

\begin{tabular}{|c|c|c|c|c|c|}
\hline Case no. & Visual loss ${ }^{\mathrm{a}}$ & Headaches & Nausea/vomiting & Lethargy & Endocrine disturbance \\
\hline 1 & Nil & 3 weeks & 3 weeks & 3 weeks & Nil \\
\hline 2 & 3 months & Nil & Nil & Nil & Short stature several years \\
\hline 3 & 1 week & 4 weeks & 4 weeks & Nil & Nil \\
\hline 4 & 2 months (few days) & 2 months & Nil & 2 months & Nil \\
\hline 5 & Nil & 6 months & 1 week & 2 months & Short stature several years \\
\hline 6 & 2 months ( 1 week) & 1 month & 2 weeks & 2 months & Polyuria/polydipsia 9 months \\
\hline 7 & 3 months (1 week) & Nil & Nil & Nil & Nil \\
\hline 8 & Nil & 2 months & 2 months & 2 months & Nil \\
\hline 9 & 3 months (2 weeks) & 2 weeks & 2 weeks & 1 month & $\begin{array}{l}\text { Polyuria/polydipsia } 2 \text { years, } \\
\text { short stature } 1 \text { year }\end{array}$ \\
\hline 10 & Nil & Several months & Nil & Several months & Nil \\
\hline
\end{tabular}

${ }^{a}$ More marked deterioration in visual function following longer-standing impairment is shown in parentheses.

A symptom or sign was recorded as present if documented as such in the case notes. A key feature was only considered absent if a specific comment to that effect was made.

\section{RESULTS}

There were 12 males and 5 females with an age range at presentation of 1-13 years and a median age of 7 years. Follow-up was available on 16 cases with a range from 2 months to 13 years and a median of 2 years.

In the group of extrinsic suprasellar tumours there were 5 craniopharyngiomas, 2 fibrillary astrocytomas, 2 teratomas and 1 germinoma. Two craniopharyngiomas and the 2 astrocytomas presented with radiological evidence of hydrocephalus. All 5 craniopharyngiomas were suprasellar in location although 2 cases had some intrasellar extension. The astrocytomas were of hypothalamic origin with extension into the third ventricle, whilst the germinoma and 2 teratomas originated from the hypothalamic and suprasellar area.

The 7 intrinsic tumours were diagnosed as gliomas on the basis of their MRI appearance. Four were unilateral and limited to the optic nerve, 2 involved both optic nerves and chiasm whilst 1 was principally a chiasmal glioma with optic nerve and tract spread. Five children were diagnosed as having neurofibromatosis type 1 .

\section{Presenting Symptoms}

The children presented with a variety of symptoms. In those with suprasellar tumours this included headaches, nausea and vomiting, lethargy, symptoms of endocrine disturbance and visual failure. The presenting symptoms and their duration are shown in Table I. In 6 of the 10 cases visual difficulty was amongst the symptoms at presentation but in only 2 was it the principal complaint. Four children had no visual symptoms at presentation. The 6 children with visual symptoms had experienced some disturbance of visual function for several months. This had been identified from a history of bumping into objects or the loss of the ability to do simple tasks and had been attributed to clumsiness. These children showed more marked visual loss in the few weeks preceding the initial assessment. In spite of the paucity of symptoms, all 10 children had reduced acuity in at least one eye, and 9 of the 10 had extensive field loss (Table IIa and b).

Table II. The ophthalmic features in children with suprasellar tumours (a) who presented with symptoms of visual failure and (b) who had no symptoms of visual failure at initial presentation

\begin{tabular}{|c|c|c|c|c|c|c|}
\hline \multirow{2}{*}{$\begin{array}{l}\text { Case } \\
\text { no. }\end{array}$} & \multirow[b]{2}{*}{ Type of tumour } & \multicolumn{2}{|c|}{ Acuity } & \multirow{2}{*}{$\begin{array}{l}\text { Visual field } \\
\text { (in better eye) }\end{array}$} & \multirow[b]{2}{*}{ Optic discs } & \multirow[b]{2}{*}{ Pupil responses } \\
\hline & & Right & Left & & & \\
\hline \multicolumn{7}{|c|}{ (a) Symptoms of visual failure at presentation } \\
\hline 2 & Craniopharyngioma & $6 / 24$ & NPL & Temporal field loss & Bilateral optic atrophy & Left RAPD \\
\hline 3 & Fibrillary astrocytoma & NPL & $6 / 12$ & Complete temporal field loss & Bilateral papilloedema & Right RAPD \\
\hline 4 & Teratoma & $\mathrm{CF}$ & NPL & Temporal field loss & Bilateral optic atrophy & Left RAPD \\
\hline 6 & Teratoma & NPL & NPL & & Bilateral optic atrophy & Unreactive pupils \\
\hline 7 & Fibrillary astrocytoma & NPL & $6 / 36$ & Nasal field loss & Bilateral optic atrophy & Right RAPD \\
\hline 9 & Germinoma & PL & $6 / 36$ & Small residual nasal field & Bilateral papilloedema & Right RAPD \\
\hline \multicolumn{7}{|c|}{ (b) No symptoms of visual failure at presentation } \\
\hline 1 & Craniopharyngioma & $6 / 24$ & $6 / 12$ & Bitemporal hemianopia & Papilloedema & Right RAPD \\
\hline 5 & Craniopharyngioma & $6 / 12$ & $6 / 9$ & $\begin{array}{l}\text { Asymmetric binasal loss with } \\
\text { enlarged blind spots }\end{array}$ & Papilloedema & Right RAPD \\
\hline $8^{\mathrm{a}}$ & Craniopharyngioma & $6 / 6$ & $1 / 60$ & Right probable temporal loss & Left disc pallor & Left RAPD \\
\hline $10^{\mathrm{a}}$ & Craniopharyngioma & $\mathrm{HM}$ & $6 / 6$ & Left supero-temporal loss ${ }^{b}$ & $\begin{array}{l}\text { Bilateral disc pallor, right } \\
\text { greater than left }\end{array}$ & Right RAPD \\
\hline
\end{tabular}

RAPD, relative afferent pupillary defect.

${ }^{a}$ Data for cases 8 and 10 refer to the features detected during routine review when the child attended with an asymptomatic loss in visual function.

${ }^{\mathrm{b}}$ Visual fields in case 10 are based on changes documented in sequential field tests performed during the 18 months prior to the onset of profound visual loss. 
Table III. The presenting features in children with optic gliomas

\begin{tabular}{lccllll}
\hline \multirow{2}{*}{$\begin{array}{l}\text { Case } \\
\text { no. }\end{array}$} & \multicolumn{2}{c}{ Acuity } & & & \\
\cline { 2 - 3 } & Right & Left & & Optic discs & Pupil responses & Presenting symptoms \\
\hline 11 & $6 / 9$ & $6 / 12$ & & NAD & NAD & Asymptomatic \\
12 & $6 / 6$ & $2 / 60$ & & Left swollen disc & Left RAPD & Visual loss and proptosis \\
13 & PL & $6 / 6$ & & Right optic atrophy & Right RAPD & Visual loss \\
14 & $3 / 60$ & $6 / 6$ & & Right swollen disc & Right RAPD & Visual loss and proptosis \\
15 & $6 / 6$ & $6 / 9$ & & Left early optic atrophy & Left RAPD & Visual loss \\
16 & $6 / 18$ & $6 / 9$ & & Right swollen disc & Right RAPD & Visual loss and proptosis \\
17 & $6 / 36$ & $1 / 60$ & & Bilateral optic atrophy & Left RAPD & Visual loss \\
\hline
\end{tabular}

Cases 11-16 had gliomas that were primarily unilateral, affecting the eye with lower acuity. Case 17 had a chiasmalglioma with optic nerve and tract spread.

Headache was the presenting symptom in the 4 children in whom there were no symptoms of visual failure and was a significant presenting feature in a total of 8 cases. It had generally been present for several weeks to months and was always accompanied by other symptoms such as nausea and vomiting or pronounced lethargy. Five of the 8 children with headache had no radiological evidence of hydrocephalus whilst headache was a presenting symptom in 3 of the 4 children with hydrocephalus.

Endocrine disturbance was not seen as the sole presenting symptom but long-standing polyuria and polydipsia was seen in 2 children. Some degree of growth failure with a longer history of short stature was found in 3 children.

In the children with intrinsic nerve tumours the presenting features were visual loss in 3 cases and 3 cases of visual loss associated with proptosis. One child with neurofibromatosis was referred with a scan showing evidence of an optic nerve glioma but was ophthalmologically asymptomatic. Three of the 6 children with symptoms of visual loss were identified and referred from community-based screening programmes.

\section{Presenting Ophthalmic Signs}

We looked at the degree of visual loss in the children with suprasellar tumours who presented with and without symptoms of visual failure.

In the 6 children with symptoms there had been loss of acuity to either perception of light (PL) or no perception of light (NPL) in at least one eye. Two of these children also had profound loss in the other eye, with acuity of NPL (case 6) and counting fingers (case 4). In the other 4 children the less affected eye saw at best $6 / 12$ and at worst $6 / 36$. The better eye also showed visual field loss ranging from an incomplete hemianopia to a small residual nasal island of vision (Table IIa).

Four children with suprasellar tumours had no visual symptoms at initial presentation. At this stage, however, cases 1 and 5 had both loss of acuity and considerable field loss and both went on to develop further visual loss in association with other more marked neurological signs. Case 8 had a normal ophthalmological examination on presentation although went on to develop an asymptomatic loss of acuity in one eye to $1 / 60$ with a suspicion of a temporal field defect in the other eye. Case 10 presented with headaches and lethargy but no ophthalmic abnormality and underwent a transsphenoidal decompression of a craniopharyngioma. $\mathrm{He}$ then developed a bilateral supero-temporal field deficit which showed little change over 18 months of follow-up until there was a marked drop in acuity in one eye to hand movements. He remained visually asymptomatic throughout (Table IIb).

Optic disc assessment and pupillary responses in children with suprasellar tumours revealed an abnormality in all cases. There were 4 cases of papilloedema (associated with obstructive hydrocephalus) and 6 of optic atrophy. Nine children had a relative afferent pupillary defect on the side with poorer acuity and 1 had bilateral unreactive pupils (Table IIa and b).

The children with gliomas had reduced acuity in one eye at presentation, despite radiological evidence in 2 cases of the tumour affecting both optic nerves. In cases 11-16 the presenting acuity ranged from $6 / 9$ to $P L$ in the affected eye whilst the vision in the other eye ranged from $6 / 6$ to $6 / 9$ with a full field. Case 17 had a chiasmal glioma with involvement of both optic nerves and tracts and reduced acuity in both eyes at $6 / 36$ and $1 / 60$. In the group with gliomas there was 1 child with normal discs, 3 with optic atrophy and 3 (those with visual loss and proptosis) with disc swelling. There was a relative pupillary defect in the 6 eyes with abnormal discs (Table III).

\section{Effect of Neurosurgical Decompression}

All 10 children with suprasellar tumours underwent neurosurgical decompression and 9 of these had documented pre- and post-operative acuities and fields (Table IV).

All 5 children with craniopharyngioma underwent a craniotomy for an attempted total excision of the tumour and this was followed by routine radiotherapy as part of the protocol for treatment. At the time of writing 3 children had completed their course of treatment whilst 2 were about to undergo their radiotherapy. Three children had a stereotactic aspiration of the cyst prior to the craniotomy and 1 
Table IV. Changes in visual acuity and fields following neurosurgical decompression in children with suprasellar tumours

\begin{tabular}{|c|c|c|c|c|c|c|}
\hline \multirow[b]{2}{*}{ Case } & \multicolumn{2}{|c|}{ Worst acuity } & \multirow[b]{2}{*}{ Worst fields } & \multicolumn{2}{|c|}{ Best acuity } & \multirow[b]{2}{*}{ Best fields } \\
\hline & Right & Left & & Right & Left & \\
\hline 1 & $6 / 36$ & $6 / 12$ & Bitemporal hemianopia & $6 / 18$ & $6 / 9$ & Little change \\
\hline 3 & NPL & $6 / 12$ & Complete temporal loss & NPL & $6 / 5$ & Little change \\
\hline 4 & $\mathrm{CF}$ & NPL & Right temporal loss & $6 / 12$ & $6 / 36$ & Right full/left residual small nasal island \\
\hline 5 & $6 / 12$ & $6 / 9$ & $\begin{array}{l}\text { Asymmetric binasal loss with } \\
\text { enlarged blind spots }\end{array}$ & $6 / 9$ & $6 / 6$ & Right temporal loss \\
\hline 6 & NPL & NPL & & $2 / 60$ & $6 / 18$ & Left homonymous hemianopia \\
\hline 7 & NPL & $6 / 36$ & Left nasal loss & $\mathrm{CF}$ & $6 / 5$ & $\begin{array}{l}\text { Right residual small temporal island, left } \\
\text { temporal loss }\end{array}$ \\
\hline 8 & $6 / 6$ & $1 / 60$ & Right probable temporal loss & $6 / 6$ & $\mathrm{C} / \mathrm{F}$ & Right full field \\
\hline 9 & PL & $6 / 36$ & Left residual nasal island & $\mathrm{CF}$ & $4 / 60$ & $\begin{array}{l}\text { Right residual small nasal island, left } \\
\text { increase in nasal field }\end{array}$ \\
\hline 10 & $\mathrm{HM}$ & $6 / 6$ & Left supero-temporal loss & $\mathrm{CF}$ & $6 / 6$ & Little change \\
\hline
\end{tabular}

Worst acuities and fields are generally immediately pre-operative whilst best findings are the best post-operative measurements during follow-up.

had a trans-sphenoidal decompression which had been performed before the introduction of the operative protocol. All 5 of the other children also had a craniotomy and 4 had additional radiotherapy. The 2 children with teratomas also had chemotherapy.

In most cases there was an improvement in acuity after surgery although the amount was variable. Three children showed a marked improvement from NPL, in 1 case to 6/18, whilst case 9 showed improvement in one eye but a subsequent drop in the other eye (presumed to be due to extension of her germinoma). Cases 1, 3, 5 and 7 showed an improvement in Snellen acuity of up to 6 lines, whilst cases 8 and 10 showed little change.

There was also some improvement in visual fields. Five children showed improvement, 3 showed no change and 1 showed a change in the pattern of field loss but no marked improvement. Fig. 1 shows the post-operative fields of case 6 who had a vision of NPL in both eyes pre-operatively. The fields show considerable improvement that was limited, however, by extensive perioperative bleeding and cerebral infarction.

In the 7 children who had completed their radiotherapy there was no evidence of radiation-induced chiasmal necrosis at the time of this study.

In cases of intrinsic nerve tumours neurosurgical decompression is not an option, but 2 of our 7 cases underwent some form of treatment. Case 14 required removal of an intra-orbital optic nerve glioma following radiological and clinical evidence of an increase in tumour size whilst case 17 required radiotherapy for a large chiasmal glioma.

\section{DISCUSSION}

A wide range of tumours were seen in these children. The suprasellar lesions consisted of a heterogeneous group of tumours, all with distinct pathological characteristics but with the common feature of a close anatomical relationship with the chiasm. Suprasellar tumours can produce the classic chiasmal field defect of a bitemporal depression, but different patterns of field loss occur depending on the relative involvement of the optic nerve, chiasm or tract. ${ }^{3,4}$ Whilst it is therefore difficult to draw conclusions regarding the pattern of field loss in such a sample, the degree of visual loss at presentation was marked in most cases and confirmed both our suspicions and previously documented findings., 5

The reason why children do not present until visual failure is so marked is uncertain. One possible reason is that other symptoms may predominate. In case 4 headache and lethargy following minor head trauma dominated the presenting picture. Similarly in the 2 girls there was emphasis on the symptoms of polyuria and polydipsia. Such non-specific symptoms may not arouse the parents' suspicion of serious pathology and it may also be that the child experiences difficulty in describing and expressing the degree of severity of such symptoms.

Signs of visual deterioration may be misinterpreted, such as the observation that the child had become clumsy in the preceding few months. Several patients had attended the optician because of concern about vision without any abnormality being detected. In these cases, acuity must have been normal and the visual difficulties due to field defects. One patient was referred to a child psychologist with a diagnosis of functional blindness. (Two children were also referred with a diagnosis of functional polydipsia.)

It may also be that children (or parents) will adapt and tolerate visual failure up to a certain lower level of visual function. From our results of presenting acuities this appears to be at a profoundly reduced level from normal, with only one seeing eye with an acuity of $6 / 12$ or less.

Two children (cases 8 and 10) demonstrated that the delayed presentation of visual symptoms is not limited to children who have been previously fit and well. These 2 children had already been diagnosed with a craniopharyngioma and had monitoring and follow-up arrangements in hand. There had also 


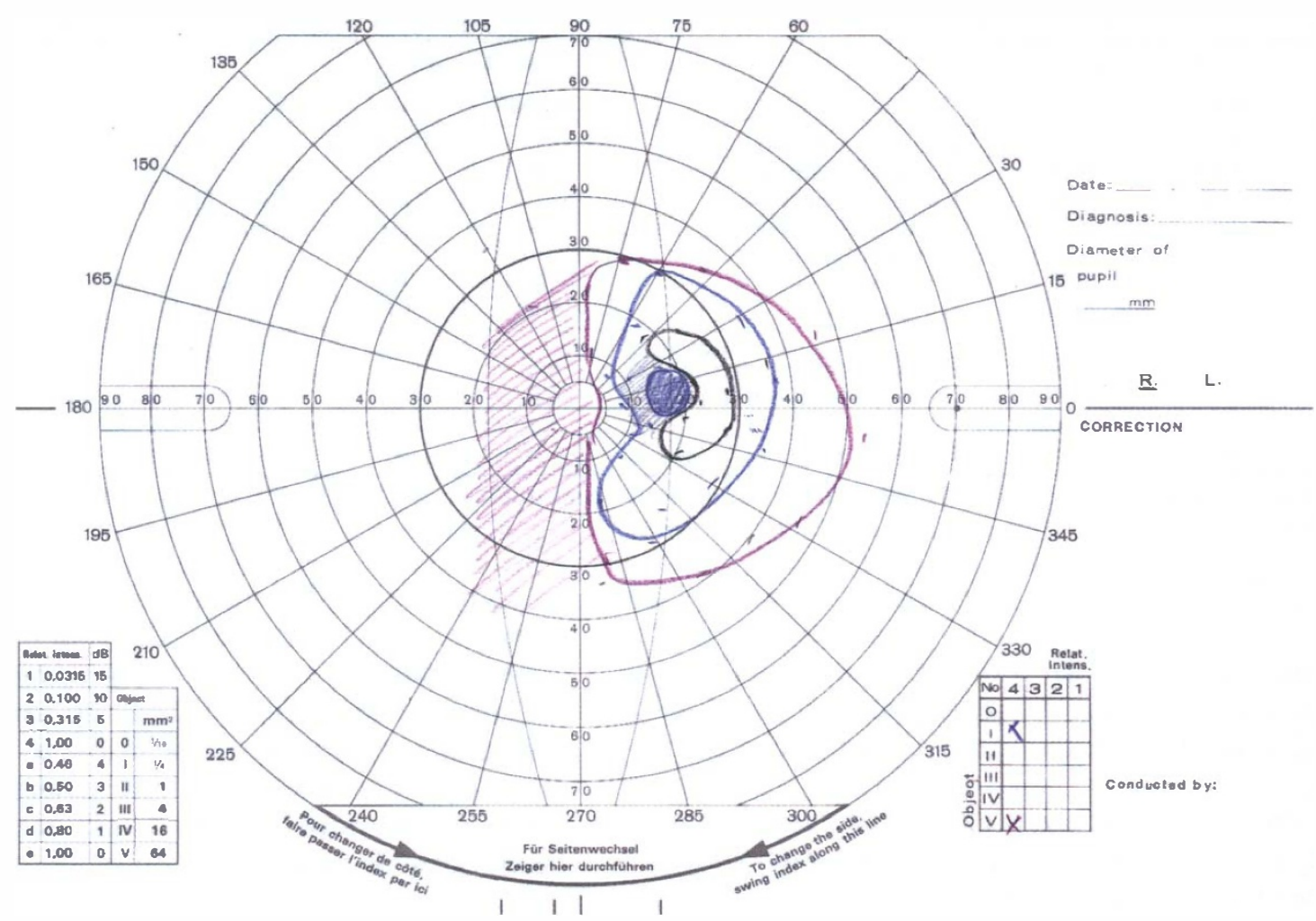

(a)

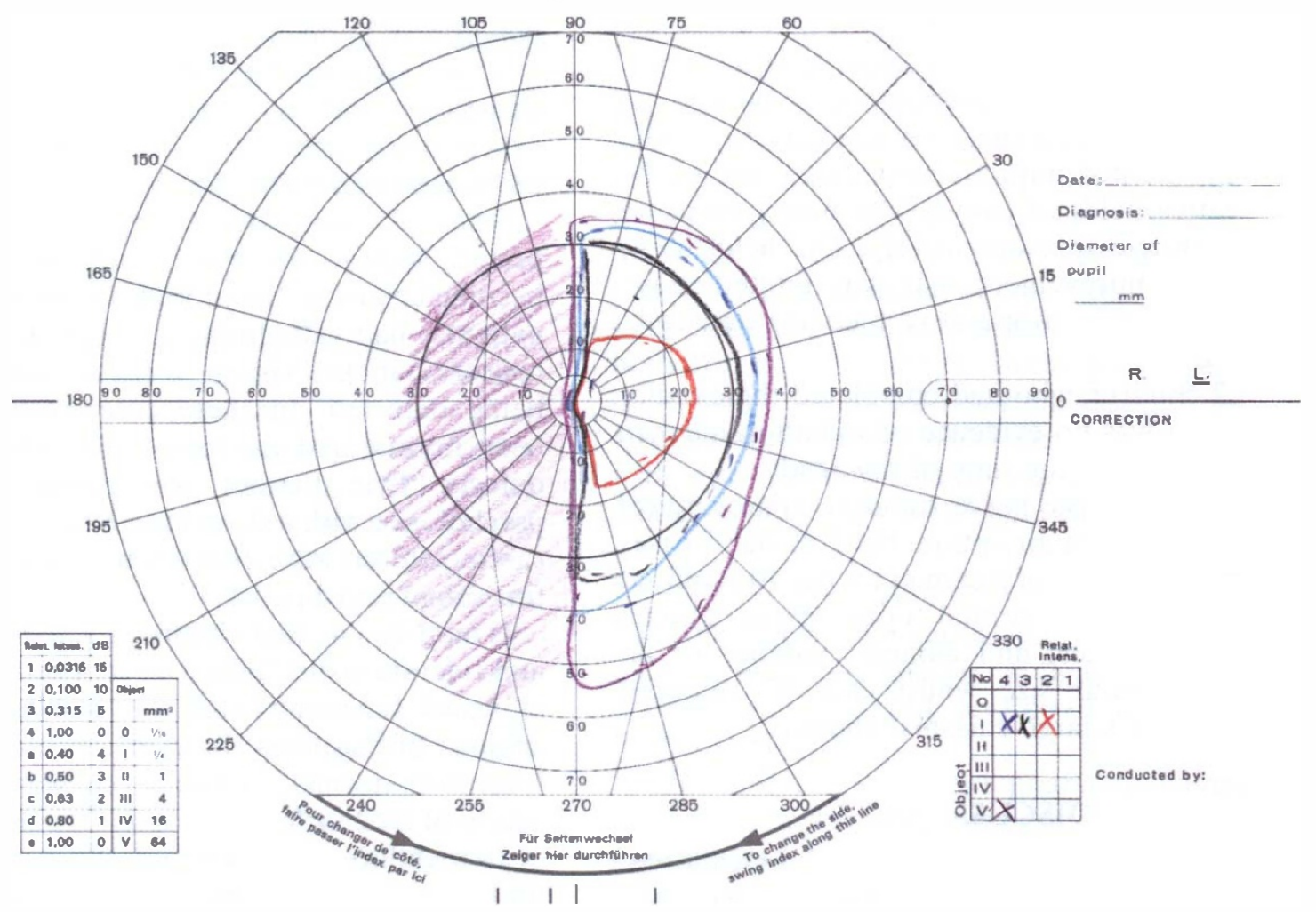

(b)

Fig. 1. The post-operative fields in case 6 following her craniotomy for a teratoma. Pre-operative acuity was no perception of light in both eyes and extensive bleeding occurred during surgery limiting recovery. 
been an explanation given to both the parents and the child regarding potential visual problems. Despite this they presented at routine review, 2 and 4 months respectively after their previous visit, with an asymptomatic but significant visual loss.

The significance of headaches in the presentation of children with brain tumours has been well documented. In a retrospective review of over 3000 children with brain tumours headache was rarely an isolated symptom and was almost always associated with other neurological symptoms. ${ }^{7}$ In addition in $20 \%$ of children over the age of 5 years with supratentorial tumours the headache had been present for over 1 year at the time of presentation. In our study headaches were always found in conjunction with other symptoms and generally had been present for weeks to months.

Once children with tumours affecting the anterior visual pathways have been identified a coordinated multi-specialist approach is valuable. ${ }^{8}$ The recurrent nature of these lesions means that follow-up and monitoring of these patients is particularly important and several children required additional intervention during the course of follow-up. Ophthalmological assessment can be performed using standard clinical tests and we found these to be effective in identifying children with both established and progressive pathology. In addition it was possible to perform a useful visual assessment even in seriously ill children. In 5 of the 10 children with suprasellar tumours deterioration in visual function was detected by such tests during routine follow-up and was a reliable indicator for more urgent neuroradiological reassessment and often subsequent neurosurgical intervention.

The mechanism of visual failure in the group with extrinsic compressive lesions has a multifactorial pathology. In astrocytomas there is a more complicated picture with several factors including infiltration and compression playing a part in the disturbance and damage of neighbouring tissues. Craniotomy for biopsy may be accompanied by some degree of debulking of the tumour but the degree of actual decompression is difficult to ascertain. The compressive effects seen in craniopharyngiomas and encapsulated tumours such as teratomas are more defined although lesions close to the third ventricle may also exert some of their pathological effects by elevating intracranial pressure. Compression leads to blockage of axonal transport and demyelination whilst raised intracranial pressure predisposes to damage by compression, with ischaemia also contributing towards visual failure. ${ }^{9}$ Infarction and axonal destruction will leave permanent damage but several of our patients presumably with total conduction block regained useful vision following decompression. Studies on chronic optic nerve compression in cats have shown that remyelination occurs after relieving the compression and this may be part of the mechanism in humans. ${ }^{10}$ Whatever the explanation, however, it is clear that rapid decompression can in certain circumstances offer the recovery of some useful vision.

Nine of the 10 children with extrinsic tumours underwent or were scheduled to undergo radiotherapy. This is an important mode of treatment in all the tumour types in this group. The treatment of craniopharyngiomas is controversial and beyond the scope of discussion here, but most studies recommend some combination of surgical excision and radiotherapy. Radiotherapy is, however, subject to several complications including endocrine deficiency, the development of secondary tumours and frontal lobe damage, with deleterious effects on higher cortical and psychomotor function. ${ }^{6,11,12}$ Specific radiation-induced damage to the optic nerve and chiasm can also occur. Chiasmal necrosis has a distinct pattern of presentation, with a latent period of 1-5 years (peak 18 months) following radiotherapy before subsequent visual loss, which tends to progress in a stepwise fashion. Diagnosis can be difficult and is largely one of exclusion, although MRI with gadolinium enhancement has been helpful in improving the recognition of this entity. ${ }^{4,13}$ In our series there was no evidence of chiasmal necrosis, although in view of its latent period it may still occur in certain patients.

Optic nerve gliomas have a different clinical course and decompression is not a treatment option. Visual loss is usually unilateral but again it was shown to be rarely symptomatic. Five of the 7 children presented with impaired vision, of whom 4 had a profound loss of acuity. Two of these cases were in young infants, and here an inability to communicate may be a contributory factor in late presentation. In a large review of all published cases of visual pathway gliomas $88 \%$ presented with loss of vision and in over half of these the presenting acuity is recorded as lower than $6 / 60 .^{14}$

It might be expected that the presence of neurofibromatosis may allow a higher index of suspicion and earlier referral. In our study, however, this appeared to occur in only 2 of the 5 children with neurofibromatosis. The remaining cases were picked up with already significantly impaired vision via the orthoptic screening service, a school medical examination or an optician.

\section{CONCLUSION}

We feel that children's complaints or parents observations of decreased visual function need full investigation. It is also important to consider the presence of any other related symptom that would increase suspicion. We found standard clinical tests 
effective in determining the extent of damage, and once diagnosed these children need a coordinated follow-up with neurosurgeons, paediatricians and oncologists where necessary. Finally it should not be forgotten that even children with no perception of light and compressive extrinsic lesions can regain useful vision if their optic nerves are speedily decompressed.

Key words: Visual failure, Suprasellar tumours, Children, Optic nerve glioma.

\section{REFERENCES}

1. Brett EM. Intracranial and spinal cord tumours. In: Brett EM, editor. Paediatric neurology. Edinburgh: Churchill Livingstone, 1983:430-61.

2. Allen ED, Byrd SE, Darling CF, Tomita T, Wilczynski MA. The clinical and radiological evaluation of primary brain tumors in children. Clinical evaluation. J Nat Med Assoc 1993;85:445-51.

3. Miller NR. Neuro-ophthalmologic topographic diagnosis of tumours and related conditions. In: Walsh and Hoyt's clinical neuro-ophthalmology. 4th ed. Baltimore: Williams \& Wilkins, 1988:1137-242.

4. Gittinger JW. Chiasmal disorders. In: Albert DM, Jakobiec FA, editors. Principles and practice of ophthalmology. Philadelphia: WB Saunders, 1994: 2615-29.
5. Miller NR. Tumours of maldevelopmental origin and related lesions. In: Walsh and Hoyt's clinical neuroophthalmology. 4th ed. Baltimore: Williams \& Wilkins, 1988:1380-425.

6. Sanford RA, Muhlbauer MS. Craniopharyngioma in children. Neurol Clin 1991;9:453-65.

7. The Childhood Brain Tumour Consortium. The epidemiology of headache among children with brain tumour. J Neuro-Oncol 1991;10:31-46.

8. Stevens MCG, Hockley AD, Spooner D, Walsh AR. Treatment for children with brain tumours. BMJ 1995;311:1213-4.

9. Miller NP. Retrobulbar compressive optic neuropathies without optic disc swelling. In: Walsh and Hoyt's clinical neuro-ophthalmology. 4th ed. Baltimore: Williams \& Wilkins, 1982:284-8.

10. Kayan A, Earl CJ. Compressive lesions of the optic nerves and chiasm: pattern of recovery of vision following surgical treatment. Brain 1975;98:13-28.

11. Hetelekidis S, Barnes PD, Tao ML, Fischer EG, Schneider L, Scott RM, Tarbell NJ. Twenty-year experience in childhood craniopharyngioma. Int $\mathrm{J}$ Radiat Oncol Biol Phys 1993;27:189-95.

12. Rajan B, Ashley S, Gorman C, Jose CC, Horwicj A, Bloom HJG, et al. Craniopharyngioma: long-term results following limited surgery and radiotherapy. Radiother Oncol 1993;26:1-10.

13. Burde RM, Savino PJ, Trobe JD. Clinical decisions in neuro-ophthalmology. 2nd ed. St Louis: Mosby-Year Book, 1992.

14. Dutton JJ. Gliomas of the anterior visual pathway. Surv Ophthalmol 1994;38:427-52. 